\title{
Lasers for every organ
}

\author{
T. R. Herrmann • A. J. Gross
}

Received: 2 March 2010/Accepted: 4 March 2010/Published online: 14 March 2010

(C) Springer-Verlag 2010

"Lasers for every urological organ?" was the working title for this issue of the World Journal of Urology, and we were able to gather contributions for almost all of them.

The godparent of endourology, Arthur Smith-together with Cinman and Andonian-shares his rich experience in the percutaneous approach to the kidney and explains the importance of lasers in this respect. By far, the most experience has been gathered with Ho:YAG lasers in stones, upper tract malignancies, foreign bodies, diverticula and upper ureter pathologies. Nd:YAG lasers have an established place in the history of lasers, and the Thulium:Yag laser is well worth observation.

Another grand senior of endourology, Demetrius Bagley, together with his highly reputed co-author Michael Grasso III, reviews and evaluates data on lasers in the treatment of upper tract malignancies. In summary, they see local laser treatment as suitable in highly selected cases that have to be followed up because of the high recurrence rate of around $60 \%$. Jean-Nicolas Cornu, from the Olivier Traxer group, came to the same conclusion with his data on 35 patients.

Thorsten Bach gathered opinions from leaders from around the world in the use of Thulium:YAG $2 \mathrm{~mm} \mathrm{cw}$ lasers in transurethral prostatectomy to bring regularity in procedural methods and terminology on this device. This is a valuable contribution towards avoiding further confusion.
There is a paucity of data on the value of lasers in the treatment of urethral strictures. Markus Bader reviews the literature on this topic, and it becomes clear that extremely variable power levels are used for the treatment of strictures. This ranges between 10 and 50 Watts. The timing of postoperative insertion of a Foley catheter differs widely, from 1 to 21 days. Recurrence of strictures lies between 0 and $20 \%$. The original data of Feng Guo in this issue of the World Journal of Urology is a valuable contribution as with 238 patients he has the largest patient numbers published on this topic to date, despite the short follow-up period.

Lasers in difficult situations are tested in vivo by Jens Bedke. With a Ho:YAG on different power settings, he tests the ability of this device to fragment foreign bodies in the urinary tract. His results are important to know, when faced with the problems of forgotten stents, an impacted catheter and such. Finally, Felix Wezel introduces an ex-vivo study on porcine kidneys using diode lasers with different wavelengths. He reports higher tissue penetration compared to the GreenLight HPS laser, which might explain the improved hemostasis. In connection with other papers, this deep penetration may be responsible for some severe side effects in diode laser use.

In conclusion, we hope that you will find this issue a helpful aid to your own judgement on lasers in urology.
T. R. Herrmann $(\bowtie)$

Department of Urology and Urological Oncology,

Hannover Medical School, Hannover, Germany

e-mail: Herrmann.Thomas@mh-hannover.de

\section{A. J. Gross}

Department of Urology, Asklepios Hospital Barmbek, Hamburg, Germany 